\title{
Unusual Event Detection Using Mean Feature Point Matching Algorithm
}

\author{
Chitra Hegde, Shakti Singh Chundawat, Divya S N \\ Department of Computer Science, Amrita Vishwa Vidyapeetham University, Mysuru Campus \\ Karnataka, India
}

\begin{abstract}
Article Info
Article history:

Received Feb 15, 2016

Revised May 18, 2016

Accepted May 29, 2016

\section{Keyword:}

Event detection

Speeded-up robust features

Unusual events

Video mining

ABSTRACT

Analysis and detection of unusual events in public and private surveillance system is a complex task. Detecting unusual events in surveillance video requires the appropriate definition of similarity between events. The key goal of the proposed system is to detect behaviours or actions that can be considered as anomalies. Since suspicious events differ from domain to domain, it remains a challenge to detect those events in major domains such as airport, super malls, educational institutions etc. The proposed Mean Feature Point Matching (MFPM) algorithm is used for detecting unusual events. The Speeded-Up Robust Features (SURF) method is used for feature extraction. The MFPM algorithm compares the feature points of the input image with the mean feature points of trained dataset. The experimental result shows that the proposed system is efficient and accurate for wide variety of surveillance videos.
\end{abstract}

Copyright () 2016 Institute of Advanced Engineering and Science. All rights reserved.

\section{Corresponding Author:}

Chitra Hegde,

Department of Computer Science,

Amrita Vishwa Vidyapeetham University, Mysuru Campus,

$114,7^{\text {th }}$ Cross, Bogadi $2^{\text {nd }}$ Stage, Mysuru-570026.

Email: chit.hegde@gmail.com

\section{INTRODUCTION}

Monitoring public or private sites has become a big issue including the monitoring of railway stations, airports, hyper markets, education institutes and inaccessible or dangerous environments. Recent technological developments have been adopted by current surveillance systems to produce fully digital video recording to keep track of suspicious events. Surveillance cameras are a great way to provide security for home or workplace. They provide us with video footage of any events which may happen, and also act as a visible deterrent to criminals. But processing of these videos remains challenging forever. Since suspicious events differ from domain to domain, it remains challenging to detect those events in each domain like airport, super malls, education institutes etc.

Unusual event can be defined as an event which deviates from normal behaviour. They occur very rarely in entire video sequence. These events are unpredictable as well. Unusual event may indicate important objects and events in wide variety of domains.

One of the major difficulties in surveillance video analysis is the huge amount of data, where only a small portion of video contains important information. The event detection in video [1]-[7] is an important task when we really focus on security issues of an organization where every single action should be considered for the detection process.

In current surveillance system, it needs human observer to assess the video that is being generated. Monitoring all the generated videos and finding the suspicious event is tedious job. For example, assuming that a rare action is related human activity, where a person is using cell phone in the place where its usage is 
prohibited. In order to overcome the drawbacks of traditional system, a new technique is proposed. This makes the way to reduce the use of man power by eliminating the need of human observer.

Unusual event recognition [8]-[11] is a challenging task due to many reasons such as confusion in actions, ambiguity in defining the normality etc. There are many existing algorithms to detect the unusual events.

Yue Zhou, Shuicheng Yan, Thomas S.Huang presented a supervised algorithm [12] for computing the similarity of motion trajectories using parameters of edit distance. The anomaly detection was considered as general outlier detection problem. There was scope to extend the algorithm for better utilization of feature space. Deepak et al. developed model for detection of dominant behaviour in videos [13] using unsupervised probabilistic topic models. They calculated normalized likelihood measures. But it is not efficient when there is no or less anomaly in the data set.

Author Peng et al. proposed a pattern mining approach [14] which utilize the patterns to address the key problems in video mining and understanding field. The Hidden Markov Model (HMM) [15] representation of object trajectories enables the similarity measures between video events by cross likelihood, but suffered from the over fitting problem due to data shortage. Fan Jiang and team [16] proposed a DHC (Dynamic Hierarchical Clustering) approach, where the HMMs are trained on many different samples and the initial clustering errors caused by over fitting are corrected in the iterative process.

Confident-Frame-based Recognizing algorithm (CFR) [17] was proposed to recognize the human activity, where high confidence video frames are used as a specialized model in the classification of the rest of the video frames. For activities such as Fighting and Running where the GMM classifiers have low detection rates.

The reviewed studies are analysed based on five aspects - surveillance target, anomaly definitions and assumptions, the feature extraction processes, learning methodologies, and modelling algorithms. The suspicious events differ from domain to domain and remains challenging to detect those events in each domain like airport, super malls, educational departments so on. Therefore the proposed system overcomes the drawback of existing system by implementing Mean Feature Point Matching (MFPM) algorithm. The SURF descriptor is used for the feature extraction [18].

\section{RESEARCH METHOD}

\subsection{System Overview}

The system takes videos from surveillance camera as input, detects the unusual events using trained dataset. The SURF technique is applied on enormous set of sample images which are considered as unusual events. Consider cell phone usage as an unusual event in prohibited area, sample images of people using cell phones in different situations are taken to train the system. Figure 1 shows few sample images that are considered for training the system. The system detect feature points in each sample image and extract feature descriptors at the interest points as shown in the Figure 2.
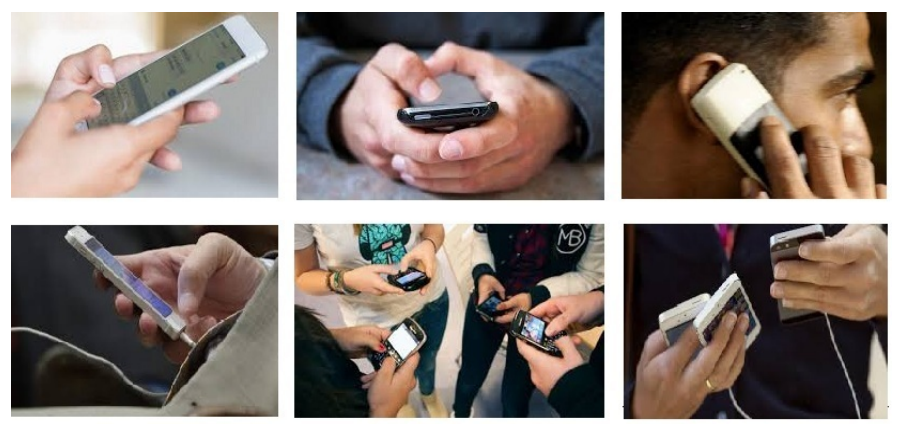

Figure 1. Sample Images 

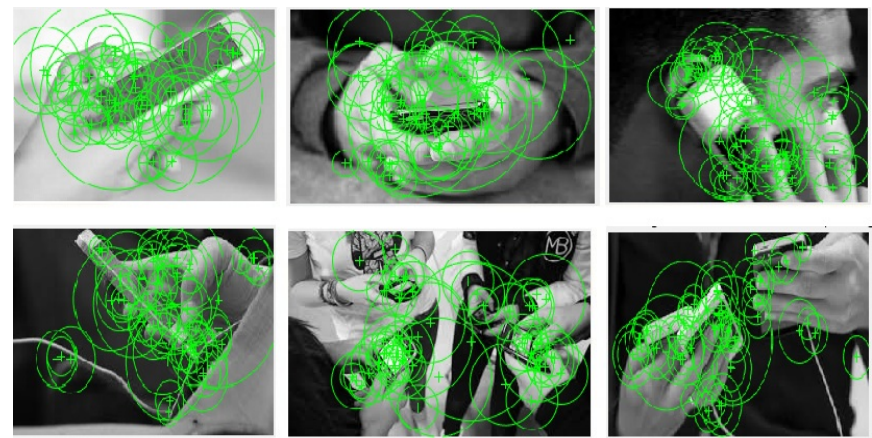

Figure 2. Visualization of strongest SURF points

The system is designed in such a way that it considers 150 strong feature points in each sample image, the mean feature point is computed and stored for further processing. The Mean Feature Point Matching (MFPM) algorithm is applied on input frames for detecting the unusual events with the help of trained samples.

\subsection{Feature Extraction}

To detect blob features, Speeded-Up Robust Features (SURF) method is implemented. The SURF method is a scale and rotation invariant interest point detector and descriptor. SURF uses a Hessian matrix which is a second derivative matrix for feature extraction.

For feature description, The SURF algorithm uses wavelet responses in horizontal and vertical directions. A neighbourhood of size $\mathrm{MxN}$ is taken around the key point. Further it is divided into sub regions. For each sub region, horizontal and vertical wavelet responses are taken and a vector $\mathrm{V}$ is formed as

$$
\mathrm{V}=\left(\sum \mathrm{d}_{\mathrm{x}}, \sum \mathrm{d}_{\mathrm{y}}, \sum\left|\mathrm{d}_{\mathrm{x}}\right|, \sum\left|\mathrm{d}_{\mathrm{y}}\right|\right)
$$

The sums of $d_{x}$ and $\left|d_{x}\right|$ are computed separately for $d_{y}<0$ and $d_{y} \geq 0$ and the sums of $d_{y}$ and $\left|d_{y}\right|$ are split up according to the sign of $\mathrm{d}_{\mathrm{x}}$, then it doubles the number of feature points.

The feature points extracted from sample images will be in the form of MxN matrix. This matrix will be converted into single dimension array for further processing by computing the mean of the feature points. The mean feature point is the average value in each column of the MxN feature matrix. The computed results will be considered for the detection of unusual events.

\subsection{Mean Feature Point Matching (MFPM) Algorithm}

The MFPM algorithm takes input in the form of video. It converts the video into set of image frames and removes the duplicate frames. The input image frames converted into grayscale images for further processing.

The desired output is to obtain a match of frame from the trained dataset. The key functionality lies in observing mean points and patterns in each frame and studying the resemblance with the trained data. Training of the dataset are stored for distinct frame, each one representing various test cases. The algorithm runs in a non-deterministic polynomial time as there is no guarantee that frame matches with the trained sets, more possible cases of trained data is taken in order to strengthen the algorithm. The following parameters are used in the algorithm.

The input video $\sum$ is converted into $\mathrm{N}_{\mathrm{f}}$ image frames, where $\mathrm{F}_{0}$ be the initial frame. Each $\mathrm{F}_{\mathrm{i}}$ where $\mathrm{i}$ $<\mathrm{N}_{\mathrm{f}}$ is converted into quadrants $\mathrm{Q}_{\mathrm{i}}\left(\mathrm{Q}_{1}, \mathrm{Q}_{2}, \mathrm{Q}_{3}, \mathrm{Q}_{4}\right)$. Each quadrant is considered for the matching process. Trained dataset will have $\mathrm{N}_{t}$ number of objects, where $\mathrm{T}_{0}$ is the initial object. Feature points $\mathrm{O}_{\mathrm{i}}$ of each $\mathrm{Q}_{\mathrm{i}}$ for all the $\mathrm{F}_{\mathrm{i}}$ is compared with the mean feature point $\mathrm{Obj}_{\mathrm{i}}$ from the trained dataset. If match found, value of corresponding $\mathrm{M}\left[\mathrm{Obj}_{\mathrm{i}}, \mathrm{Q}_{\mathrm{i}}\right]$ will become 1 and count $\mathrm{C}$ will increased by 1 . Ratio is calculated by dividing the count $\mathrm{C}$ by total number of frames $\mathrm{N}_{\mathrm{f}}$. The loop continues till the end of the sequence. The algorithm returns the matrix as a result. The Feature Matching (FM) matrix $\mathrm{M}\left[\mathrm{Obj}_{\mathrm{i}}, \mathrm{Q}_{\mathrm{i}}\right]$ represent the mapping between the objects in the trained dataset and each quadrant of the input frame. 
Table 1. Parameters used in MFPM Algorithm

\begin{tabular}{cl}
\hline Parameters & Meaning \\
\hline $\mathrm{F}_{0}$ & Input video \\
$\mathrm{N}_{\mathrm{f}}$ & Total number of frames \\
$\mathrm{T}_{0}$ & Initial frame in trained data set \\
$\mathrm{N}_{\mathrm{t}}$ & Total number of trained data set \\
$\mathrm{Q}_{\mathrm{i}}$ & Quadrants of input frame \\
$\mathrm{Obj}_{\mathrm{i}}$ & Mean feature points of object in trained data \\
$\mathrm{O}_{\mathrm{i}}$ & Feature points of $\mathrm{Q}_{\mathrm{i}}$ \\
$\mathrm{R}$ & Ratio of matching \\
$\mathrm{C}$ & Total number of matches \\
$\mathrm{M}\left[\mathrm{Obj} \mathrm{j}_{\mathrm{i}}, \mathrm{Q}_{\mathrm{i}}\right]$ & Feature Matching (FM) matrix \\
\hline
\end{tabular}

MFPM $\left(\sum, \mathrm{F}_{0}, \mathrm{~N}_{\mathrm{f}}, \mathrm{T}_{0}, \mathrm{~N}_{\mathrm{t}}, \mathrm{Q}_{\mathrm{i}}, \mathrm{O}_{\mathrm{i}}, \mathrm{M}\left[\mathrm{Obj}_{\mathrm{i}}, \mathrm{Q}_{\mathrm{i}}\right], \mathrm{R}, \mathrm{C}\right)$
step1: Input the sequence $\sum$
step2: Preprocess $\sum$
step3:
step4: Monsider the initial frame $\mathrm{F}_{0}$
step5: Each $\mathrm{F}_{\mathrm{i}}$ is segmented into quadrants $\mathrm{Q}_{\mathrm{i}}$
step6: Compare $\mathrm{O}_{\mathrm{i}}$ of $\mathrm{F}_{\mathrm{i}}$ with $\mathrm{Obj}_{\mathrm{i}}$ in
the trained dataset $\mathrm{T}_{0}$ to $\mathrm{T}_{\mathrm{i}}$ where $\mathrm{i}<\mathrm{N}_{\mathrm{t}}$
step7: Each entry corresponding to $\mathrm{Q}_{\mathrm{i}}$ of the $\mathrm{F}_{\mathrm{i}}$ and
$\quad$ Obj $\mathrm{j}_{\mathrm{i}}$ of the $\mathrm{T}_{\mathrm{i}}$ is plotted in $\mathrm{M}\left[\mathrm{Obj}_{\mathrm{i}}, \mathrm{Q}_{\mathrm{i}}\right]$
step8: $\mathrm{M}\left[\mathrm{Obj}_{\mathrm{i}}, \mathrm{Q}_{\mathrm{i}}\right]=\left\{\begin{array}{l}1, \text { if } \mathrm{O}_{\mathrm{i}} \text { is matched with } \mathrm{Obj}_{\mathrm{i}} \\ 0, \text { otherwise }\end{array}\right.$
step9: If $\mathrm{M}\left[\mathrm{Obj}_{\mathrm{i}}, \mathrm{Q}_{\mathrm{i}}\right]$ value 1, then $\mathrm{C} \rightarrow \mathrm{C}+1$
step10: Ratio $\mathrm{R} \rightarrow \mathrm{C} / \mathrm{N}_{\mathrm{f}}$
step1 1: Return matrix $\mathrm{M}\left[\mathrm{Obj}_{\mathrm{i}}, \mathrm{Q}_{\mathrm{i}}\right]$

Figure 3. MFPM Algorithm

\section{RESULTS AND ANALYSIS}

The proposed system consider enormous image samples to extract the features of interested area. The testing is performed by giving the captured videos as input. The system is able to detect cell phone usage. Figure 4 (a) shows the sample image where the cell phone is detected in the first quadrant. Figure 5 (a) shows the FM matrix which indicates that the sixth object in the trained dataset is matching with the object in the first quadrant of the current input image. Figure 4 (d) shows the sample image where the cell phone is detected in the second quadrant, hence other quadrants are not checked further. Once the objects are matched, it will move to the next frame in the sequence. Thus it will reduce the time complexity. 


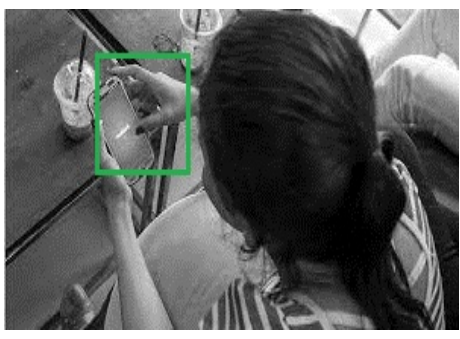

(a)

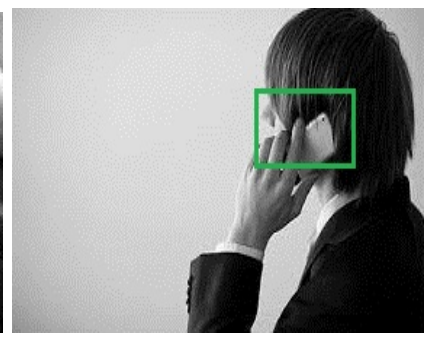

(b)

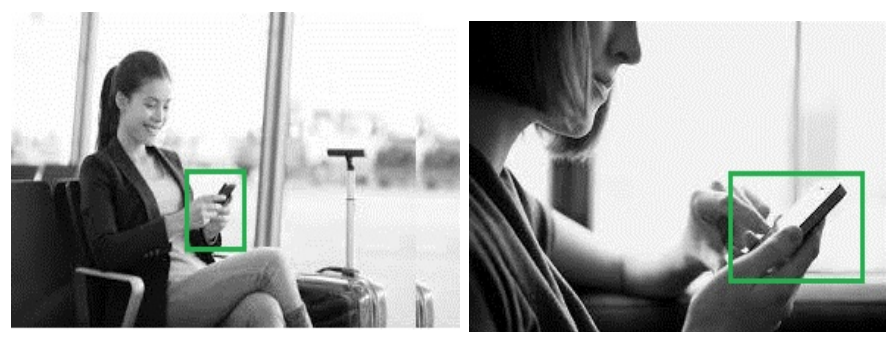

(c)

(d)

Figure 4. Unusual event detected in different images

The count variable $\mathrm{C}$ is maintained throughout the program. At the end, $\mathrm{C}$ value indicate the number of frames with unusual event in the input video.

\begin{tabular}{|c|c|c|c|c|c|}
\hline & $\mathrm{Q}_{1}$ & $\mathrm{Q}_{2}$ & $\mathrm{Q}_{3}$ & $\mathrm{Q}_{4}$ & Count \\
\hline $\mathrm{Obj}_{0}$ & 0 & 0 & 0 & 0 & 0 \\
\hline $\mathrm{Obj}_{1}$ & 0 & 0 & 0 & 0 & 0 \\
\hline $\mathrm{Obj}_{2}$ & 0 & 0 & 0 & 0 & 0 \\
\hline $\mathrm{Obj}_{3}$ & 0 & 0 & 0 & 0 & 0 \\
\hline $\mathrm{Obj}_{4}$ & 0 & 0 & 0 & 0 & 0 \\
\hline $\mathrm{Obj}_{5}$ & 0 & 0 & 0 & 0 & 0 \\
\hline $\mathrm{Obj}_{6}$ & 1 & - & - & - & 1 \\
\hline
\end{tabular}

(a)

\begin{tabular}{|c|c|c|c|c|c|}
\hline & $\mathrm{Q}_{1}$ & $\mathrm{Q}_{2}$ & $\mathrm{Q}_{3}$ & $\mathrm{Q}_{4}$ & Count \\
\hline $\mathrm{Obj}_{0}$ & 0 & 0 & 0 & 0 & 0 \\
\hline $\mathrm{Obj}_{1}$ & 0 & 0 & 0 & 0 & 0 \\
\hline $\mathrm{Obj}_{2}$ & 0 & 0 & 0 & 0 & 0 \\
\hline $\mathrm{Obj}_{3}$ & 0 & 0 & 0 & 0 & 0 \\
\hline $\mathrm{Obj}_{4}$ & 0 & 0 & 0 & 0 & 0 \\
\hline $\mathrm{Obj}_{5}$ & 0 & 0 & 0 & 0 & 0 \\
\hline $\mathrm{Obj}_{6}$ & 0 & 0 & 0 & 0 & 0 \\
\hline $\mathrm{Obj}_{7}$ & 0 & 0 & 0 & 0 & 0 \\
\hline $\mathrm{Obj}_{8}$ & 0 & 0 & 0 & 0 & 0 \\
\hline $\mathrm{Obj}_{9}$ & 0 & 0 & 0 & 0 & 0 \\
\hline $\mathrm{Obj}_{10}$ & 0 & 0 & 1 & - & 1 \\
\hline
\end{tabular}

(c)

\begin{tabular}{|c|c|c|c|c|c|}
\hline & $Q_{1}$ & $Q_{2}$ & $Q_{3}$ & $Q_{4}$ & Count \\
\hline Objo & 0 & 0 & 0 & 0 & 0 \\
\hline$O b_{1}$ & 0 & 0 & 0 & 0 & 0 \\
\hline$O b_{j} 2$ & 0 & 0 & 0 & 0 & 0 \\
\hline$O b_{j} j_{3}$ & 0 & 1 & - & - & 1 \\
\hline
\end{tabular}

(b)

\begin{tabular}{|c|c|c|c|c|c|}
\hline & $\mathrm{Q}_{1}$ & $\mathrm{Q}_{2}$ & $\mathrm{Q}_{3}$ & $\mathrm{Q}_{4}$ & Count \\
\hline $\mathrm{Obj}_{0}$ & 0 & 0 & 0 & 0 & 0 \\
\hline $\mathrm{Obj}_{1}$ & 0 & 0 & 0 & 0 & 0 \\
\hline $\mathrm{Obj}_{2}$ & 0 & 0 & 0 & 0 & 0 \\
\hline $\mathrm{Obj}_{3}$ & 0 & 0 & 0 & 0 & 0 \\
\hline $\mathrm{Obj}_{4}$ & 0 & 0 & 0 & 0 & 0 \\
\hline $\mathrm{Obj}_{5}$ & 0 & 0 & 0 & 0 & 0 \\
\hline$:$ & $:$ & $:$ & $:$ & $:$ & $:$ \\
$:$ & $:$ & $:$ & $:$ & $:$ & $:$ \\
$:$ & $:$ & $:$ & $:$ & $:$ & $:$ \\
$:$ & $:$ & $:$ & $:$ & $:$ & $:$ \\
\hline $\mathrm{Obj}_{\mathrm{N}}$ & 0 & 0 & 0 & 1 & 1 \\
\hline
\end{tabular}

(d)

Figure 5. Feature Matching (FM) matrix 
To evaluate the performance of the MFPM algorithm, it is compared with the trajectory similarity analysis algorithm. Figure 6 shows the performance comprasion of MFPM algorithm using precision recall curve.
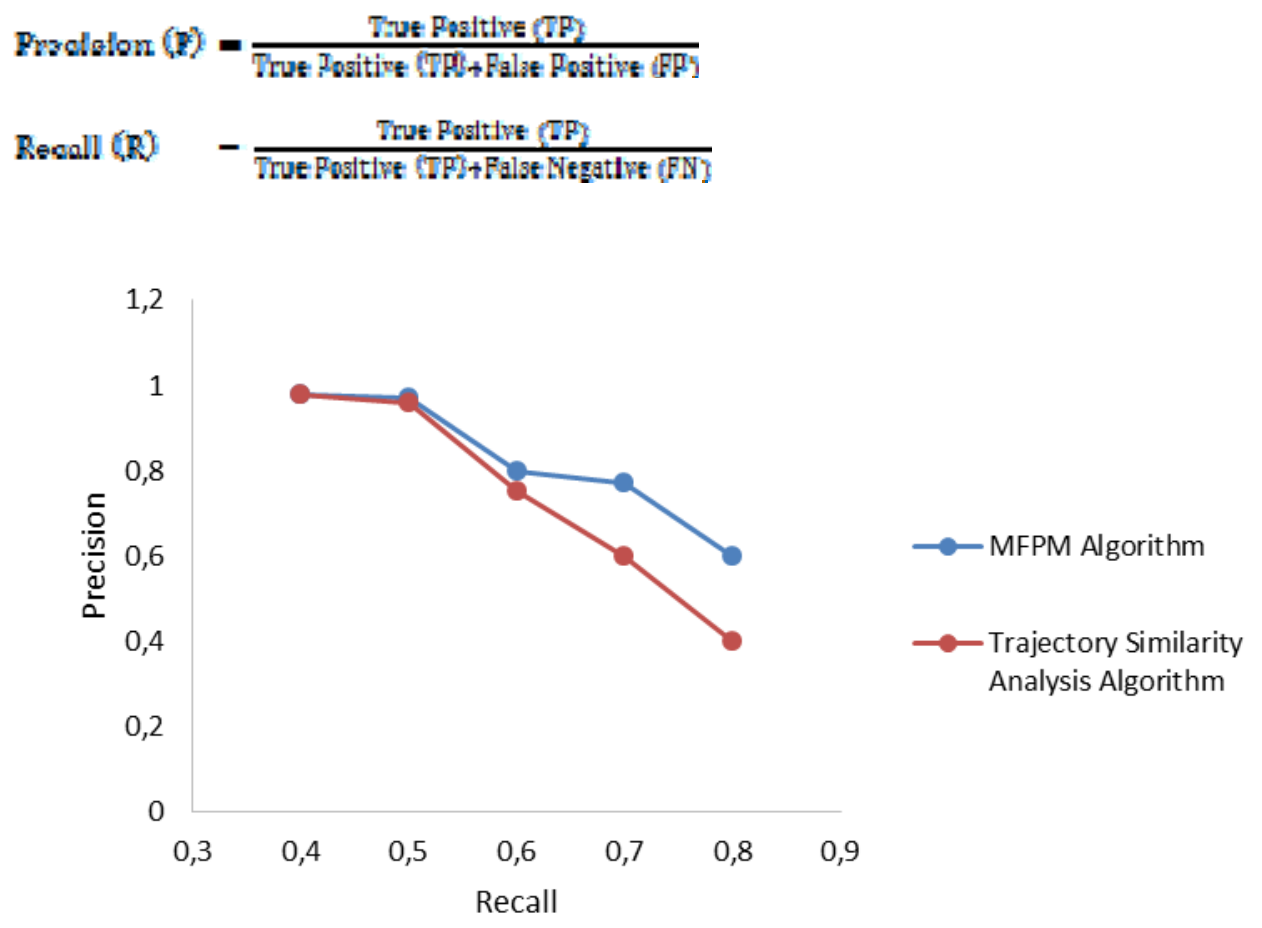

Figure 6. Precision-Recall curve

Table 2 shows the test result of the system with two different video inputs. Videos are captured in different scenarios. These videos are converted into image frames with frame rate 23 frames per second. To reduce the processing time, duplicate frames are removed. The system detected 17 image frames as frames with unusual events in the first video input. The system efficiently detecting the cell phone usage in all the scenarios. It can detect objects with unusual behaviour despite a scale change or in-plane rotation.

Table 2. Test Result

\begin{tabular}{cccc}
\hline $\begin{array}{c}\text { Sample } \\
\text { Videos }\end{array}$ & $\begin{array}{c}\text { Total number of } \\
\text { frames }\end{array}$ & $\begin{array}{c}\text { Number of frames } \\
\text { Considered }\end{array}$ & $\begin{array}{c}\text { Unusual events detected } \\
\text { (C) }\end{array}$ \\
\hline Video1 & 3895 & 779 & 17 \\
Video2 & 4352 & 870 & 6 \\
\hline
\end{tabular}

\section{CONCLUSION}

The proposed system implements the robust technique for detecting unusual events in surveillance videos. The mean feature points matching method will keep track of the unusual events and helps in taking effective actions. The experimented results shows the efficiency in detection of cell phone usage in different quadrants of the input image frame. The objects in the trained dataset are matched with input image despite a scale change or in-plane rotations. In future, additional test cases can be included in the trained dataset to achieve accurate results. The degree of rarity in events can be classified using dynamic classifiers to enhance the performance of the algorithm.

\section{REFERENCES}

[1] Bazmi A. and Faez K., "Increasing the Accuracy of Detection and Recognition in Visual Surveillance," International Journal of Electrical and Computer Engineering, vol/issue: 2(3), pp. 395, 2012.

[2] Lee K. M. and Kwon J., "A unified framework for event summarization and rare event detection," In 2012 IEEE Conference on Computer Vision and Pattern Recognition, pp. 1266-1273, 2012. 
[3] Morris B. T. and Trivedi M. M., "A survey of vision-based trajectory learning and analysis for surveillance," Circuits and Systems for Video Technology, IEEE Transactions on., vol/issue: 18(8), pp. 1114-27, 2008.

[4] Huang Z. F. and Mori G., "SFU at TRECVid 2010: Surveillance Event Detection," In TRECVID, 2010.

[5] Xia Z., et al., "PKU-NEC@ TRECVID 2012 SED: Uneven-sequence based event detection in surveillance video," In Proc. TRECVID, 2012.

[6] Chen M., et al., "Semantic event extraction using neural network ensembles," In Semantic Computing, 2007, ICSC 2007, International Conference on, pp. 575-580, 2007.

[7] Noaman R. A., et al., "Human Detection Framework for Automated Surveillance Systems," International Journal of Electrical and Computer Engineering (IJECE), vol/issue: 6(2), 2015.

[8] Zhong H., et al., "Detecting unusual activity in video," In Computer Vision and Pattern Recognition, 2004. CVPR 2004. Proceedings of the 2004 IEEE Computer Society Conference on, vol. 2, pp. II-819, 2004.

[9] Lu C., et al., "Abnormal event detection at $150 \mathrm{fps}$ in matlab," In Proceedings of the IEEE International Conference on Computer Vision, pp. 2720-2727, 2013.

[10] Sodemann A. A., et al., "A review of anomaly detection in automated surveillance," Systems, Man, and Cybernetics, Part C: Applications and Reviews, IEEE Transactions on., vol/issue: 42(6), pp. 1257-72, 2012.

[11] Nievas E. B., et al., "Violence detection in video using computer vision techniques," In Computer Analysis of Images and Patterns, pp. 332-339, 2011.

[12] Zhou Y., et al., "Detecting anomaly in videos from trajectory similarity analysis," In Multimedia and Expo, 2007 IEEE International Conference on, pp. 1087-1090, 2007.

[13] A. Mukerjee, et al., "Unsupervised Modelling of 'Usual' Events and Detecting Anomalies in Videos".

[14] Cui P., et al., "Hierarchical visual event pattern mining and its applications," Data Mining and Knowledge Discovery, vol/issue: 22(3), pp. 467-92, 2011.

[15] Zhang D., et al., "Semi-supervised adapted hmms for unusual event detection," In Computer Vision and Pattern Recognition, 2005. CVPR 2005. IEEE Computer Society Conference on, vol. 1, pp. 611-618, 2005.

[16] Jiang F., et al., "Abnormal event detection from surveillance video by dynamic hierarchical clustering," In Image Processing, 2007. ICIP 2007. IEEE International Conference on, vol. 5, pp. V-145, 2007.

[17] Lin W., et al., "Human activity recognition for video surveillance," In Circuits and Systems, 2008. ISCAS 2008. IEEE International Symposium on, pp. 2737-2740, 2008.

[18] Chandrika L., "Implementation Image Retrieval and Classification with SURF Technique," IJISET-International Journal of Innovative Science, Engineering \& Technology, vol/issue: 1(4), 2014. 